OPEN ACCESS

Edited by:

J. Luis Espinoza,

Kindai University, Japan

Reviewed by:

David W. Chan,

The University of Hong Kong,

Hong Kong

An T. T. Dao,

Ho Chi Minh City Medicine and Pharmacy University, Vietnam

*Correspondence: Rong Zhang xqpharmacylab@126.com

Specialty section:

This article was submitted to Pharmacology of Anti-Cancer Drugs,

a section of the journal

Frontiers in Pharmacology

Received: 01 January 2018 Accepted: 30 April 2018

Published: 18 May 2018

Citation:

Zhou L, Yang F, Li G, Huang J, Liu Y, Zhang Q, Tang Q, Hu C and

Zhang $R$ (2018) Coptisine Induces Apoptosis in Human Hepatoma Cells Through Activating 67-kDa Laminin

Receptor/cGMP Signaling. Front. Pharmacol. 9:517. doi: 10.3389/fphar.2018.00517

\section{Coptisine Induces Apoptosis in Human Hepatoma Cells Through Activating 67-kDa Laminin Receptor/cGMP Signaling}

\author{
Li Zhou' ${ }^{1}$, Fan Yang ${ }^{2}$, Guobing Li', Jingbin Huang ${ }^{1}$, Yali Liu', Qian Zhang', Qin Tang', \\ Changpeng $\mathrm{Hu}^{1}$ and Rong Zhang ${ }^{1 *}$
}

${ }^{1}$ Department of Pharmacy, The Second Affiliated Hospital, Third Military Medical University, Chongqing, China,

${ }^{2}$ Department of Orthopaedic, General Hospital of Tibetan Military Command Lhasa, Lhasa, China

Hepatocellular carcinoma $(\mathrm{HCC})$ is the most common primary cancer of the liver. Hence, new anti-liver cancer treatment strategies need to be urgently developed. Coptisine is a natural alkaloid extracted from rhizoma coptidis which exhibits anticancer activity in various preclinical models, including liver cancer. However, the molecular mechanisms underlying the anti-liver cancer effects of coptisine remains unclear. We used flow cytometry to assess the binding of coptisine to 67LR expressed on the surface of SMMC7721, HepG2, LO2 and H9 cells. Then SMMC7721, HepG2 and BEL7402 cells, belonging to the HCC cell lines, were treated with coptisine. The cell viability was detected using a cell counting kit-8 assay. Apoptosis was evaluated using flow cytometry and transferase-mediated dUTP nick-end labeling (TUNEL) assay. Apoptotic-related proteins and tumor death receptor 67-kDa laminin receptor (67LR) were detected using Western blot analysis. The cyclic guanosine $3^{\prime}, 5^{\prime}$-monophosphate (cGMP) concentration was determined using enzyme-linked immunosorbent assay. sh67LR lentivirus, anti67LR antibody, and cGMP inhibitor NS2028 were used to determine how a 67LR/cGMP signaling pathway regulated coptisine-induced apoptosis. Tumor growth inhibited by coptisine was confirmed in a SMMC7721 cell xenograft mouse model. Coptisine selectively exhibited cell viability in human hepatoma cells but not in normal human hepatocyte cell line LO2 cells. Coptisine promoted SMMC7721 and HepG2 cell apoptosis by increasing 67LR activity. Both 67LR antibody and sh67LR treatment blocked coptisine-induced apoptosis and inhibition of cell viability. Coptisine upregulated the expression of cGMP. Moreover, cGMP inhibitor NS2028 significantly decreased coptisine-induced apoptosis and inhibition of cell viability. In vivo experiments confirmed that coptisine could significantly suppress the tumor growth and induce apoptosis in SMMC7721 xenografts through a 67LR/cGMP pathway. Coptisinemediated 67LR activation may be a new therapeutic strategy for treating hepatic malignancy.

Keywords: apoptosis, cGMP, coptisine, 67LR, hepatocellular carcinoma 


\section{INTRODUCTION}

Hepatocellular carcinoma (HCC), one of the most common malignancies worldwide, comprises only $10-30 \%$ surgical candidates (Gravitz, 2014; Jin et al., 2017). At present, chemotherapy and some oral multikinase inhibitors are primarily used to treat intermediate-advanced HCC (Zhang et al., 2016). However, the treatment benefits are still limited, such as poor curative effects and adverse reactions ( $\mathrm{Lu}$ et al., 2013; Yoshimoto et al., 2013; Lv et al., 2015). Therefore, more effective pharmacological agents for treating HCC are urgently needed.

Coptisine, an isoquinoline alkaloid, is extracted from rhizoma coptidis. It has a variety of pharmacological effects, such as antibacterial (Kwon et al., 2008), anticachectic (Iizuka et al., 2002; Iizuka, 2016), and lipid-lowering (Kou et al., 2016). Moreover, previous studies demonstrated anticancer activities of coptisine in colorectal cancer, breast cancer, and lung cancer. Huang T. et al. (2017) discovered that coptisine suppressed HCT-116 cell-related tumor growth by promoting tumor cell apoptosis via inhibiting the RAS-ERK pathway. Another study (Rao et al., 2017) found that coptisine could significantly induce mitochondria-mediated apoptosis in non-small-cell lung cancer A549 cells. The present study indicated that coptisine selectively inhibited cell viability in human HCC cell line SMMC7721, HepG2 and BEL7402 cells but not in normal human hepatocyte cell line LO2 cells, suggesting that coptisine might be a potential cancer treatment for HCC. However, its mechanism of action still need further research (Lin et al., 2004; Huang et al., 2015).

Apoptosis is an important mode of programmed cell death. It can be regulated and performed by the activated members of caspase family, such as caspase 3 and 8 (Fan et al., 2017; Huang W. et al., 2017). Moreover, it is well known that caspase activation is initiated and propagated by two major signaling pathways: extrinsic pathway and intrinsic pathway. The extrinsic pathway refers mainly to a death receptor pathway, which is located on the cell surface. It can directly activate caspase 8 , and then the downstream caspase 3 is once activated (Li et al., 2015; Boege et al., 2017; Xu et al., 2017).

The $67-\mathrm{kDa}$ laminin receptor (67LR), a new death receptor, is a laminin-binding protein overexpressed in various types of cancer, including multiple myeloma, bile duct carcinoma, colorectal carcinoma, breast carcinoma, and cervical cancer (Song et al., 2012; Kumazoe et al., 2013a; Pesapane et al., 2017). Substantial evidence supported that 67LR regulated the sensitivity and invasiveness of cancer cells to chemotherapeutics. Moreover, recent studies focused more on the effect of 67LR on cancer cell apoptosis (Song et al., 2012; Jovanovic et al., 2015; Zhou Y. et al., 2016). Discovered published study demonstrated that polyphenol (-)-epigallocatechin-3-O-gallate (EGCG) could effectively increase apoptosis in myeloma cells after activating 67LR (Dorchies et al., 2009). The underlying mechanism included the cancer-specific cyclic guanosine $3^{\prime}, 5^{\prime}$-monophosphate (cGMP) upregulation by inhibiting the expression of phosphodiesterase 5. The cGMP upregulation could exactly be a rate-determining process of 67LR-dependent cell apoptosis. In addition, Kumazoe et al. (2013a) found that EGCG activated 67LR and then suppressed cancer stem cell properties in pancreatic ductal adenocarcinoma. In normal cells, Activation of 67LR could be an effective agent in suppressing LPS-induced retinal inflammation (Kumazoe et al., 2013a; Huang Y. et al., 2017).

In the present study, it was hypothesized that coptisine induced apoptosis in HCC through a 67LR/cGMP death receptor pathway. Lentivirus knocked-down 67LR were employed to verify the effects of coptisine on apoptosis in SMMC7721 cells in vivo and in vitro, and further investigated the underlying molecular mechanism downstream of 67LR. These findings provided a novel mechanistic basis for coptisine in HCC treatment.

\section{MATERIALS AND METHODS}

\section{Reagents}

Coptisine (purity > 98.0\% by high-performance liquid chromatography) was provided by Must Biological Technology Co., Ltd. (Chengdu, China) and solubilized in dimethylsulfoxide (DMSO). The cyclic GMP complete enzymelinked immunosorbent assay (ELISA) kit was purchased from Abcam (Burlingame, CA, United States). The NS2028 reagent was obtained from Beyotime Biotechnology (Jiangsu, China). Allophycocyanin(APC)-Annexin V products were obtained from BD Pharmingen (San Jose, CA, United States). Antibody against 67LR was purchased from Abcam. Antibodies against poly (ADP-ribose) polymerase (PARP), cleaved caspase 3, and cleaved caspase 8 were purchased from Immunoway Biotechnology Company (Jiangsu, China).

\section{Cell Culture}

SMMC7721, HepG2, BEL7402, $\mathrm{LO}_{2}$ and $\mathrm{H} 9$ cells were provided by the American Type Culture Collection (Manassas, VA, United States). The cells were cultured in Dulbecco's modified Eagle's medium (DMEM) supplemented with 10\% fetal bovine serum. They were incubated with a humidified atmosphere containing $5 \% \mathrm{CO}_{2}$ at $37^{\circ} \mathrm{C}$.

\section{Lentiviral Gene Transfer and Gene Silencing}

The human sh67LR (5'-GATCCGCCTTCACTAACCAGATCC ATTCAAGAGATGGATCTGGTTAGTGAAGGTTTTTTG-3')

and control shRNA plasmids were purchased from Genomeditech (Shanghai, China). Plasmids were co-transfected with lentiviral packaging vectors (hU6-MCS-CMV-mcherryPGK-Puro-WPRE) into 293FT cells by using Lipofectamine 3000 according to the manufacturer's protocol. Then the supernatant containing lentivirus was collected and used to infect the SMMC7721 cells. Subsequently, the cells were grown in $5 \mu \mathrm{g} / \mathrm{mL}$ puromycin for selecting the stable cell clone (verification results are shown in Supplementary Figure S1).

\section{Cell Viability Assay}

The cell viability was tested using a cell counting kit-8 (CCK-8) (Beyotime, Shanghai, China) according to the manufacturer's 
protocol to explore the effectiveness of coptisine treatment for SMMC7721, HepG2, BEL7402 cells and $\mathrm{LO}_{2}$ cells in vitro. The number of cells was measured after coptisine $(0,12.5,25,50$ and $100 \mu \mathrm{M}$ ) treatment for $24 \mathrm{~h}$. Also, the number of cells was measured after coptisine $(50 \mu \mathrm{M})$ treatment for $0,6,9,12$ and $24 \mathrm{~h}$. The supernatant of each group was discarded. The cells were then incubated in DMEM basic medium containing CCK-8 for another $2 \mathrm{~h}$ at $37^{\circ} \mathrm{C}$. An automated microplate reader (Thermo Fisher, Waltham, MA, United States) was used to read the optical density (OD) value at $450 \mathrm{~nm}$.

\section{Apoptosis Analysis}

Apoptotic cells were detected by APC-conjugated Annexin $\mathrm{V}$-APC/DAPI staining (BD Pharmingen) according to the manufacturer's protocol, and then analyzed with a FACSVantage SE Flow Cytometer (BD Biosciences, San Jose, CA, United States). Both early apoptotic (Annexin V-positive and DAPI-negative) and late apoptotic (Annexin V-positive and DAPI-positive) cells were used for determining cell death.

The apoptotic cells were also detected using an in situ cell death detection kit (TUNEL technology) (Roche, Mannheim, Germany) following the manufacturer's instructions. Images were captured using a Leica scanning confocal microscope (TCS SP5, Leica Microsystems).

\section{Flow Cytometry Analysis}

Fluorescein isothiocyanate (FITC) is one of the fluorescein commonly used for marking alkaloids. We labeled Coptisine with FITC by incubating $50 \mathrm{mM}$ of Coptisine with $0.5 \mathrm{mM}$ FITC in a $100 \mathrm{mM} \mathrm{NaHCO}$ buffer solution $(\mathrm{PH}=9.0)$ for $30 \mathrm{~min}$ at room temperature in the dark. Thereafter SMMC7721, HepG2, LO2, and H9 cells were incubated with FITC-labeled coptisine for $30 \mathrm{~min}$ and analyzed by flow cytometry in a FACS Vantage SE Flow Cytometer instrument. Heat-inactivated FITC-labeled coptisine was used as a negative control and $1 \mu \mathrm{g} / \mathrm{ml}$ PI was used to discriminate live cells. Flow cytometry data were analyzed with the FlowJo software package (Tree Star, Ashland, OR, United States).

\section{cGMP Assays}

cGMP levels in cells treated with coptisine for about $3 \mathrm{~h}$ were measured using the Cyclic GMP Complete ELISA Kit (Abcam), following the manufacturer's instructions and the OD absorbance was read at $405 \mathrm{~nm}$ using an automated microplate reader (Thermo Fisher, Waltham, MA, United States).

\section{Western Blot Analysis}

Western blot analysis was performed as previously described (Zhou L. et al., 2016). Briefly, cell samples were collected and lysed in $1 \times$ NuPAGE LDS (Lithium dodecyl sulfate) sample buffer (Invitrogen, Carlsbad, CA, United States) to obtain total protein, whose concentrations were measured using a bicinchoninic acid protein assay kit (Beyotime). Then, $30 \mu \mathrm{g}$ of sample proteins were separated using sodium dodecyl sulfate-polyacrylamide gel electrophoresis gels and transferred onto nitrocellulose membranes. Then, the membranes were blocked with $5 \%$ fat-free dry milk in $1 \times$ Tris-buffered saline including $0.05 \%$ Tween 20 and incubated with primary antibodies. The following primary antibodies were used: antiPARP (1:500), anti-Cleaved Caspase-3 (1:500), anti-Cleaved Caspase-8 (1:500) form Immunoway Biotechnology Company, JiangSu, China, anti- 67LR (1:1000) from Abcam, United States, and anti- $\beta$-actin (1:1000) from Santa Cruz Biotechnologies. After incubating with horseradish peroxidase-conjugated secondary antibodies, protein bands were detected on a bio-imaging system (Bio-Rad, Berkeley, CA, United States). The ImageJ software was used to measure the densitometric values of the bands.

\section{Immunohistochemical Analysis}

The cells were mounted on the confocal dish (NEST BD-Falcon, Corning, NY, United States). After treatment in groups, the cells were washed three times with phosphate-buffered saline (PBS), fixed with $4 \%$ paraformaldehyde for $20 \mathrm{~min}$, permeabilized using $0.1 \%$ Triton X-100 for $10 \mathrm{~min}$, and then blocked with $1 \%$ bovine serum albumin for $30 \mathrm{~min}$. Next, the cells were subsequently incubated with target antibodies at $4^{\circ} \mathrm{C}$ overnight. The following primary antibodies were used: anti-Cleaved Caspase-3 (1:500), anti-Cleaved Caspase-8 (1:500) form Immunoway Biotechnology Company, JiangSu, China, and anti- 67LR (1:1000) from Abcam. Followed by a secondary peroxidase-conjugated goat anti-mouse antibody (Molecular Probes, Invitrogen, United States) for $1 \mathrm{~h}$ at room temperature. After washing with PBS, the images were captured using a confocal laser scanning microscope (TCS SP5; Leica Microsystems).

\section{Xenograft Assay}

Male nude mice (5 weeks old) were purchased from Vital River Laboratories (Beijing, China) and fed in a pathogen-free room. All the animal studies were performed in accordance with China's animal welfare legislation for the care and use of animals and approved by the Third Military Medical University Chongqing, China. SMMC7721 cells $\left(2 \times 10^{6}\right.$ cells per mouse $)$ were subcutaneously inoculated into the right hind leg of 40 mice, which were then randomly divided into four groups ( $n=10$ per group). The other 10 mice were inoculated with sh67LR SMMC7721 cells as the fifth group. After tumor inoculation for 5 days, the mice in all five groups received normal saline, coptisine (50 mg/kg) + immunoglobulin G (IgG), coptisine + anti-67LR $(20 \mu \mathrm{g} / \mathrm{mL})$, coptisine + scramble shRNA, and coptisine + sh67LR (tail intravenous injection, five times a week) for about 6 weeks. Tumor growth and body weights were measured every week, and tumor volumes were calculated according to the formula (length $\times$ width $^{2}$ )/2. Finally, tumor tissues from representative mice in each group were sectioned, embedded in paraffin, and then processed for hematoxylin and eosin (H\&E) and immunohistochemical staining and analysis performed as previously described (Yang et al., 2017).

\section{Statistical Analyses}

Data were expressed as means \pm standard deviation and analyzed using SPSS13.0 software (SPSS, Chicago, IL, United States). A one-way analysis of variance (ANOVA) with repeated measures was used to evaluate the significance of the effect of different 
treatments of coptisine on apoptosis. The Student $t$-test and ANOVA were used for comparing two or more groups, respectively. The results were statistically significant at $P<0.05$.

\section{RESULTS}

\section{Coptisine Inhibited Cell Viability in Human HCC Cell Lines SMMC7721, HepG2 and BEL7402 Cells but Not in Normal Human Hepatocyte Cell Line LO2 Cells}

First, the effects of coptisine on cytotoxicity in human HCC SMMC7721, HepG2, BEL7402 cells and normal human hepatocyte cell line LO2 cells were evaluated using CCK-8 assay. As shown in Figures 1A,C,E, exposure of SMMC7721, HepG2 and BEL7402 cells to $12.5 \mu \mathrm{M}$ coptisine led to a moderate inhibition of cell viability, and 25,50 , and $100 \mu \mathrm{M}$ coptisine significantly exacerbated this effect. In contrast, some coptisine toxicity was observed in normal human hepatocyte cell line LO2 cells compared with these HCC cells. In addition, a time-effect analysis indicated that $50 \mu \mathrm{M}$ coptisine could decrease the viability of SMMC7721 cells slightly after $6 \mathrm{~h}$, which became more significant after 9, 12, and 24 h. However, coptisine treatment induced relatively lower toxicity in LO2 cells than in SMMC7721 cells (Figures 1B,D,F). These results suggested that coptisine selectively inhibited cell viability in human HCC SMMC7721, HepG2 and BEL7402 cells but not in normal human hepatocyte cell line LO2 cells.
A

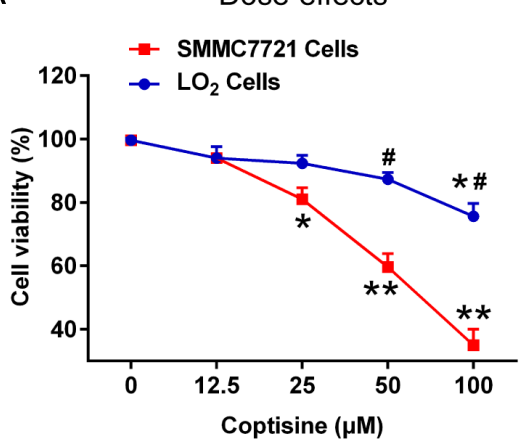

C
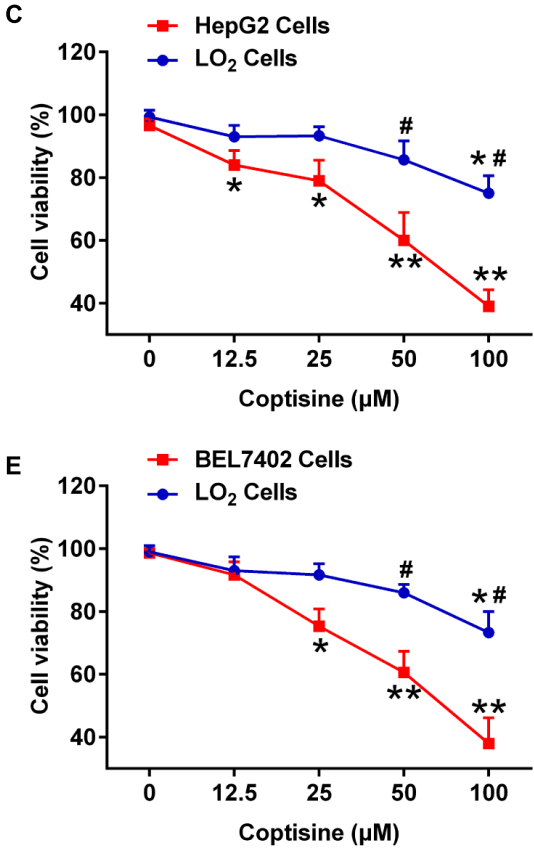

B Time-effects

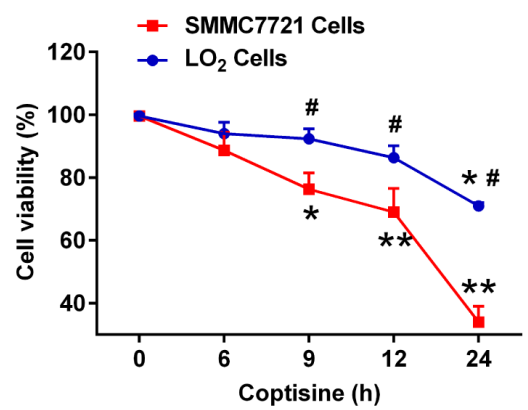

D

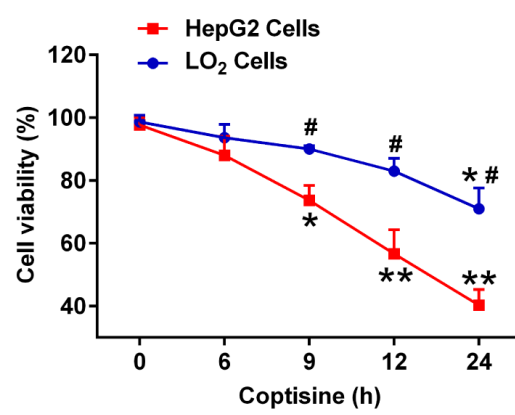

$\mathbf{F}$

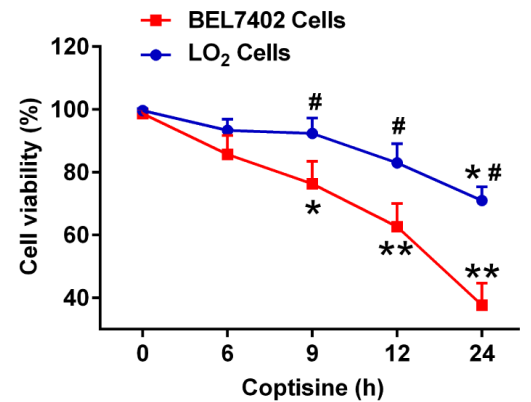

FIGURE 1 | Coptisine inhibited cell viability in SMMC7721, HepG2 and BEL7402 cells but not in LO2 cells. (A) SMMC7721 cells, (C) HepG2 cells, (E) BEL7402 cells and LO2 cells were treated with indicated concentrations of coptisine for $24 \mathrm{~h}$, and cell viability was detected using CCK-8 assay. ${ }^{*} P<0.05$ and ${ }^{* *} P<0.01$ compared with the untreated group (control) of the same cells. ${ }^{\#} P<0.05$ compared with the same operated group of SMMC7721 cells. (B) SMMC7721 cells, (D) HepG2 cells, (F) BEL7402 cells and LO2 cells were treated with $50 \mu \mathrm{M}$ coptisine for $0-24 \mathrm{~h}$, and cell viability was detected using CCK-8 assay. ${ }^{*} P<0.05$ and ${ }^{* *} P<0.01$ compared with the 0 -h group of the same cells. ${ }^{\#} P<0.05$ compared with the same group of SMMC7721 cells. The results were representative of three independent experiments. Error bars represent mean \pm SD. 


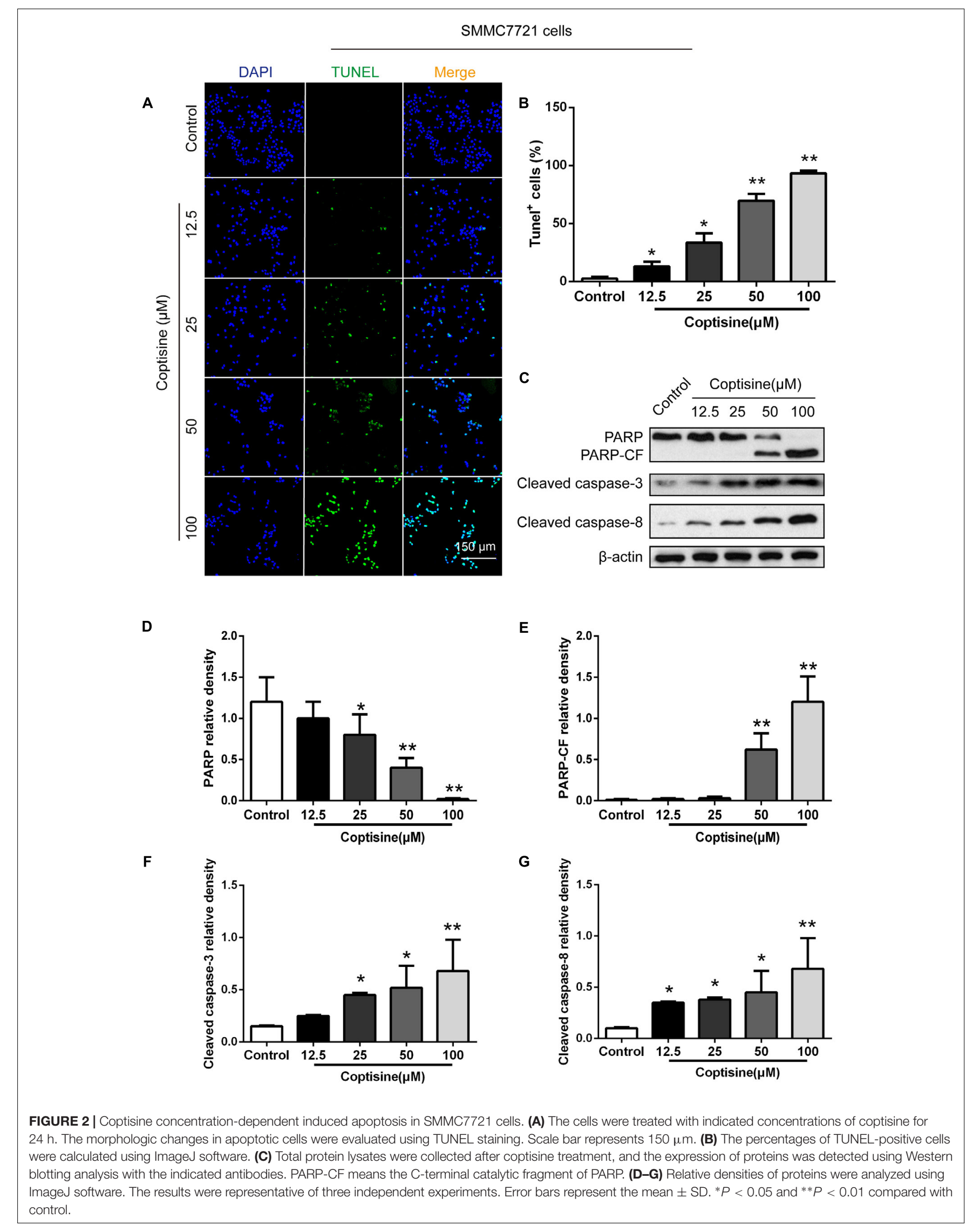


A

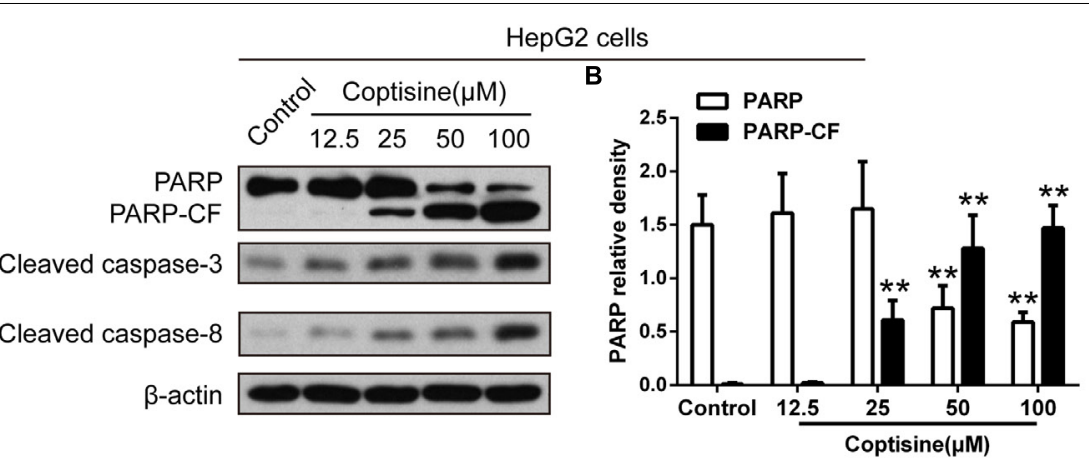

C
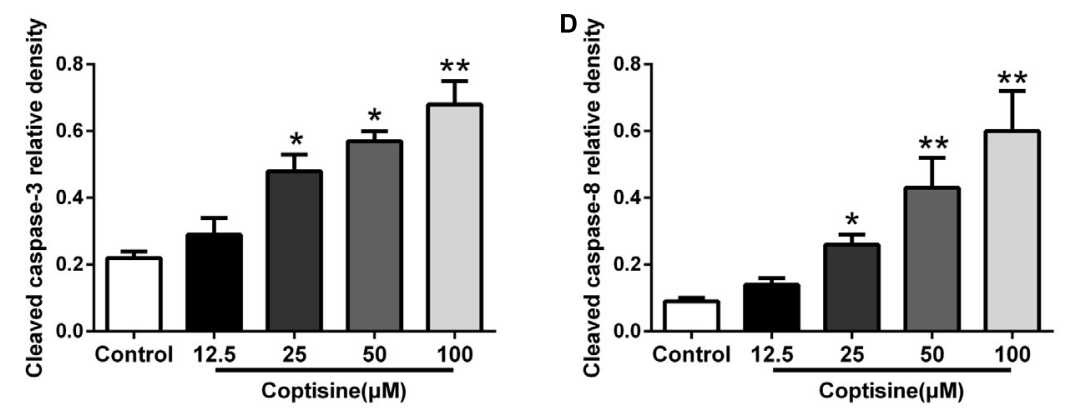

E SMMC7721 cells HepG-2 cells

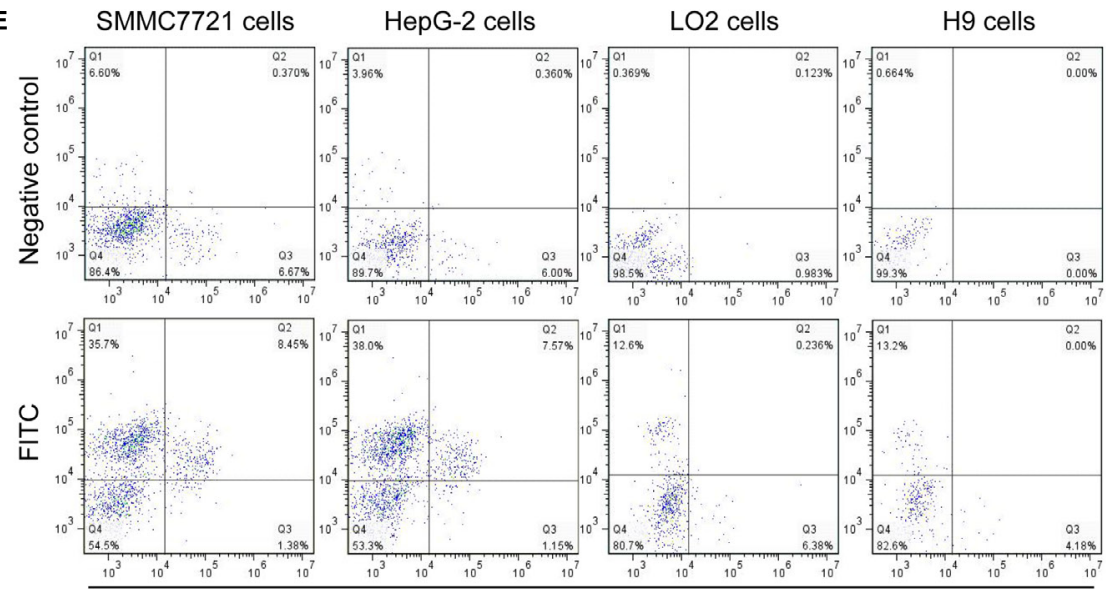

PI
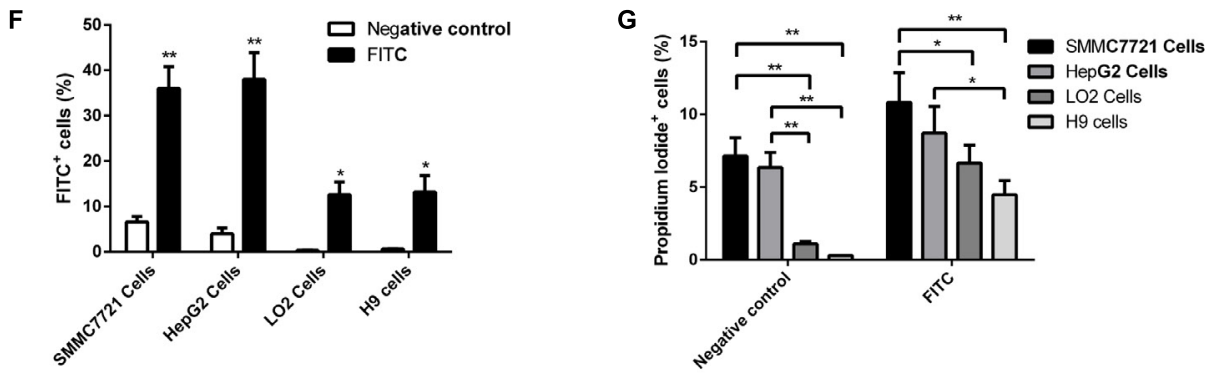

FIGURE 3 | Coptisine concentration-dependent induced apoptosis in HepG2 cells and Coptisine could bind to the surface of hepatoma carcinoma cells. (A) After HepG2 cells were treated with indicated concentrations of coptisine for $24 \mathrm{~h}$, total protein lysates were collected, and the expression of proteins was detected using Western blotting analysis with the indicated antibodies. PARP-CF means the C-terminal catalytic fragment of PARP. (B-D) Relative densities of proteins were analyzed using ImageJ software. The results were representative of three independent experiments. Error bars represent the mean $\pm S D$. ${ }^{*} P<0.05$ and ${ }^{* *} P<0.01$ compared with control. (E) $50 \mathrm{mM}$ Coptisine was labeled by $0.5 \mathrm{mM}$ FITC for $30 \mathrm{~min}$ (FITC-labeled coptisine), and then added to SMMC7721 cells, HepG2 cells, LO2 cells and H9 cells respectively. $1 \mu \mathrm{g} / \mathrm{ml} \mathrm{PI}$ was used to determine living cells. FITC-labeled coptisine was heat-inactivated as a negative control. Cells were filtrated by flow cytometry. (F) The percentages of FITC positive cells were counted using FlowJo software $(n=3)$. (G) The percentages of PI positive cells were counted $(n=3) .{ }^{*} P<0.05$ and ${ }^{* *} P<0.01$ compared with the appointed group. 
A

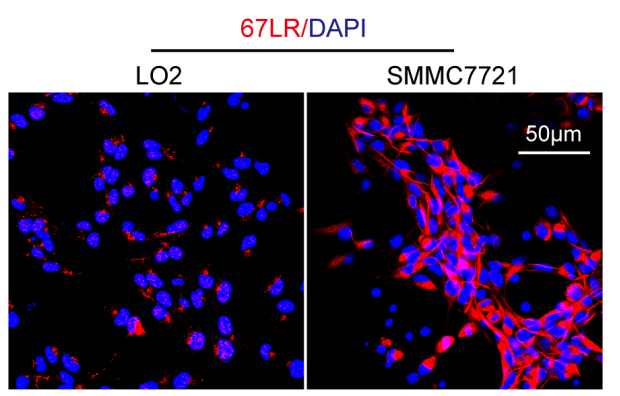

B

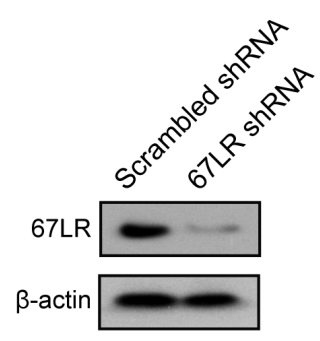

C

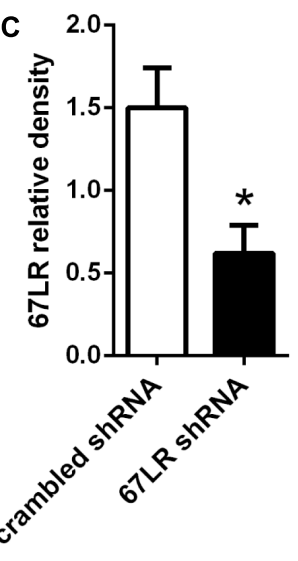

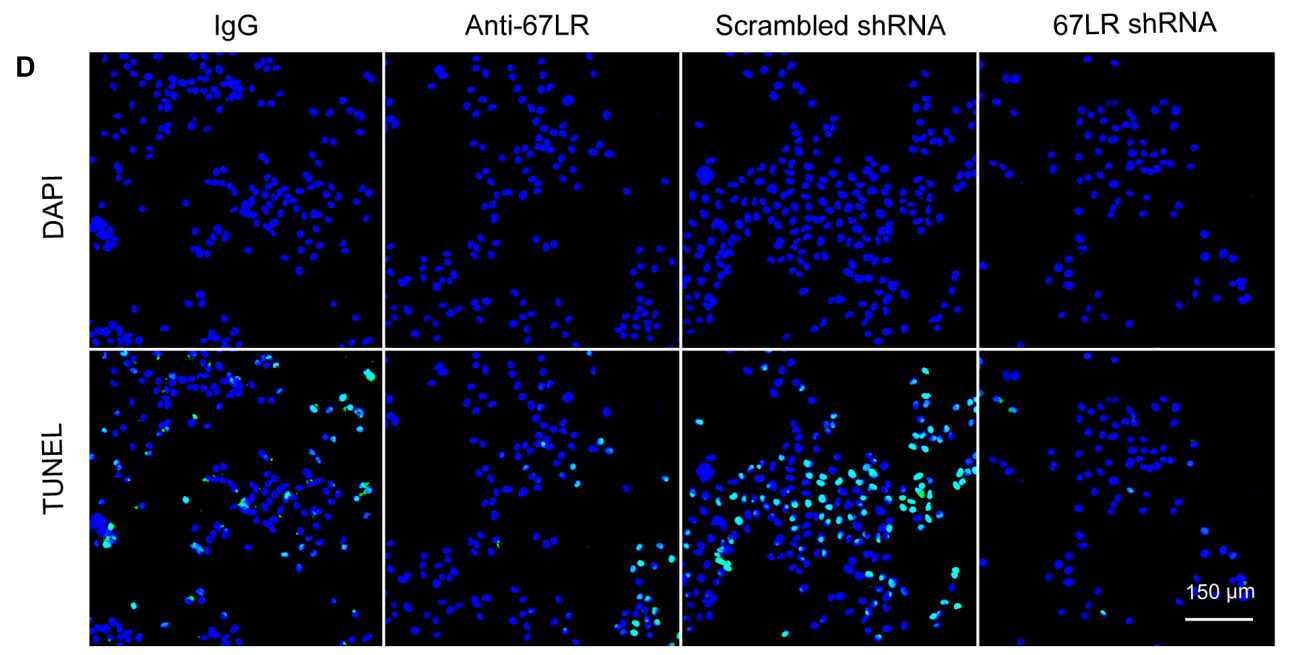

Coptisine $(50 \mu \mathrm{M})$

E

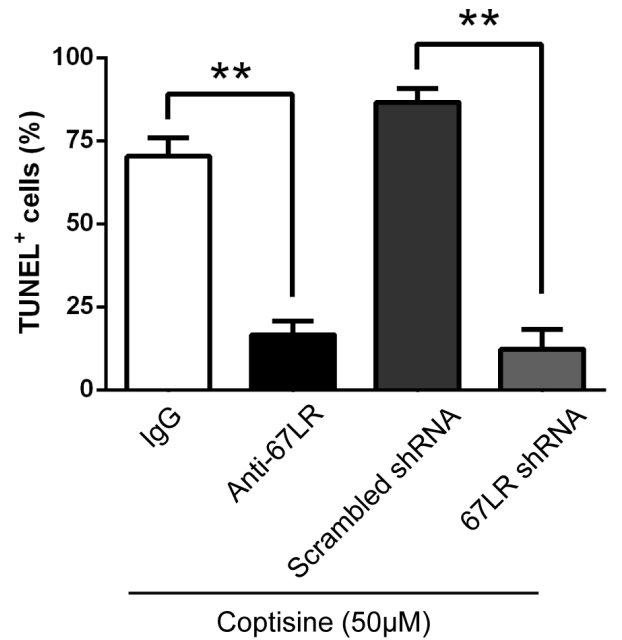

$\mathbf{F}$

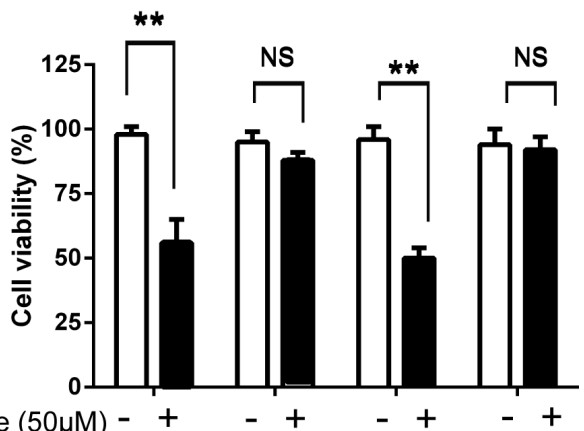

Coptisine $(50 \mu \mathrm{M})-+\quad+\quad+\quad+\quad-+$

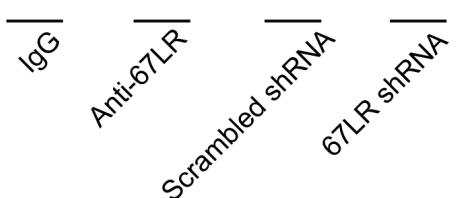

FIGURE 4 | Coptisine promoted SMMC7721 cell apoptosis by increasing 67LR activity. (A) LO2 cells and SMMC7721 cells were separately collected and stained with anti-67LR (red) and DAPI (blue) to identify the expression of 67LR using immunofluorescence. The scale bar represents $50 \mu \mathrm{m}$. (B,C) The expression of $67 \mathrm{LR}$ protein was tested in scramble shRNA SMMC7721 and sh67LR SMMC7721 cells using Western blot analysis. The relative density of 67LR expression was analyzed using ImageJ software. ${ }^{*} P<0.05$ compared with the scramble shRNA group. (D,E) Anti-67LR and sh67LR models were used to detect cell apoptosis after coptisine $(50 \mu \mathrm{M})$ treatment. Then, morphologic changes in apoptotic cells were evaluated using TUNEL staining. Scale bar represents $150 \mu \mathrm{m}$. The percentages of TUNEL-positive cells were calculated using ImageJ software. ${ }^{* *} P<0.01$. (F) Cell viability was tested using CCK-8 assay. ${ }^{* *} P<0.01$; NS means no statistical significance. The results were representative of three independent experiments. All data are represented as mean \pm SD. 


\section{Coptisine-Induced Apoptosis in SMMC7721 and HepG2 Cells}

TUNEL technology was used to study coptisine-induced apoptosis so as to explore the effect of coptisine on SMMC7721 cells. Coptisine was shown to significantly increase apoptosis of SMMC7721 cells in a dose-dependent manner (Figures 2A,B). Additionally, the expression levels of apoptotic -related proteins, including PARP and cleaved caspases 3 and 8 in SMMC7721 cells (Figures 2C-G) and HepG2 cells (Figures 3A-D) were detected by Western blot analysis. It showed that the protein expression of C-terminal catalytic fragment of PARP (PARP-CF) and cleaved caspases 3 and 8 significantly increased with the increasing dose of coptisine. Next, to assess if coptisine directly interacts with cell surface expressed 67LR, we performed flow cytometry analysis using FITC-labeled Coptisine. As shown in Figures 3E-G, Coptisine effectively binds 67LR expressed on the surface of various cell types including SMMC7721, HepG2, LO2, and $\mathrm{H} 9$ cells with apparent higher 67LR expression in malignant cells than in normal cells.

\section{Coptisine Promoted SMMC7721 and HepG2 Cells Apoptosis by Increasing 67LR Activity}

The immunofluorescence results revealed that the expression of 67LR in SMMC7721 cells was much higher than that in human hepatocyte cell line LO2 cells, which was consistent with the finding of other studies that 67LR was highly expressed in cancer cells. Kumazoe et al. (2013b) discovered that a
67LR-mediated pathway contributed significantly to cancerselective apoptosis. In this study, it was suspected that 67LR was related to the development of HCC induced by SMMC7721 cells, and might be an important target for coptisine-induced apoptosis in SMMC7721 cells. Our immunofluorescence results indicated that the expression of 67LR protein in SMMC7721 cells was significantly higher than that of LO2 cells (Figure 4A). A lentiviral vector for sh67LR (Supplementary Figure S1) was successfully constructed, and the protein expression of 67LR in SMMC7721 cells was verified after sh67LR lentivirus treatment (Figures 4B,C). Then the effects of 67LR knockdown and 67LR antibody on cell viability and apoptosis were examined after coptisine treatment. As shown in Figures 4D,E, $\mathbf{5 A}, \mathbf{B}$, both TUNEL assay and flow cytometry revealed that coptisine-induced SMMC7721 cell apoptosis decreased after being given 67LR antibody or sh67LR. The results of CCK8 assay also revealed that both 67LR antibody and sh67LR treatment blocked coptisine-induced inhibition of cell viability (Figure 4F). Moreover, similar results were confirmed in HepG2 cells by TUNEL assay (Figure 6A) and western blot analysis (Figures 6B-F). All the results indicated that coptisine induced SMMC7721 and HepG2 cells apoptosis through a 67LRdependent pathway.

\section{Coptisine Induced the cGMP Upregulation After Activating 67LR in SMMC7721 Cells}

67LR acted as a death receptor through cancer-specific cGMP upregulation. And the cGMP upregulation could exactly be

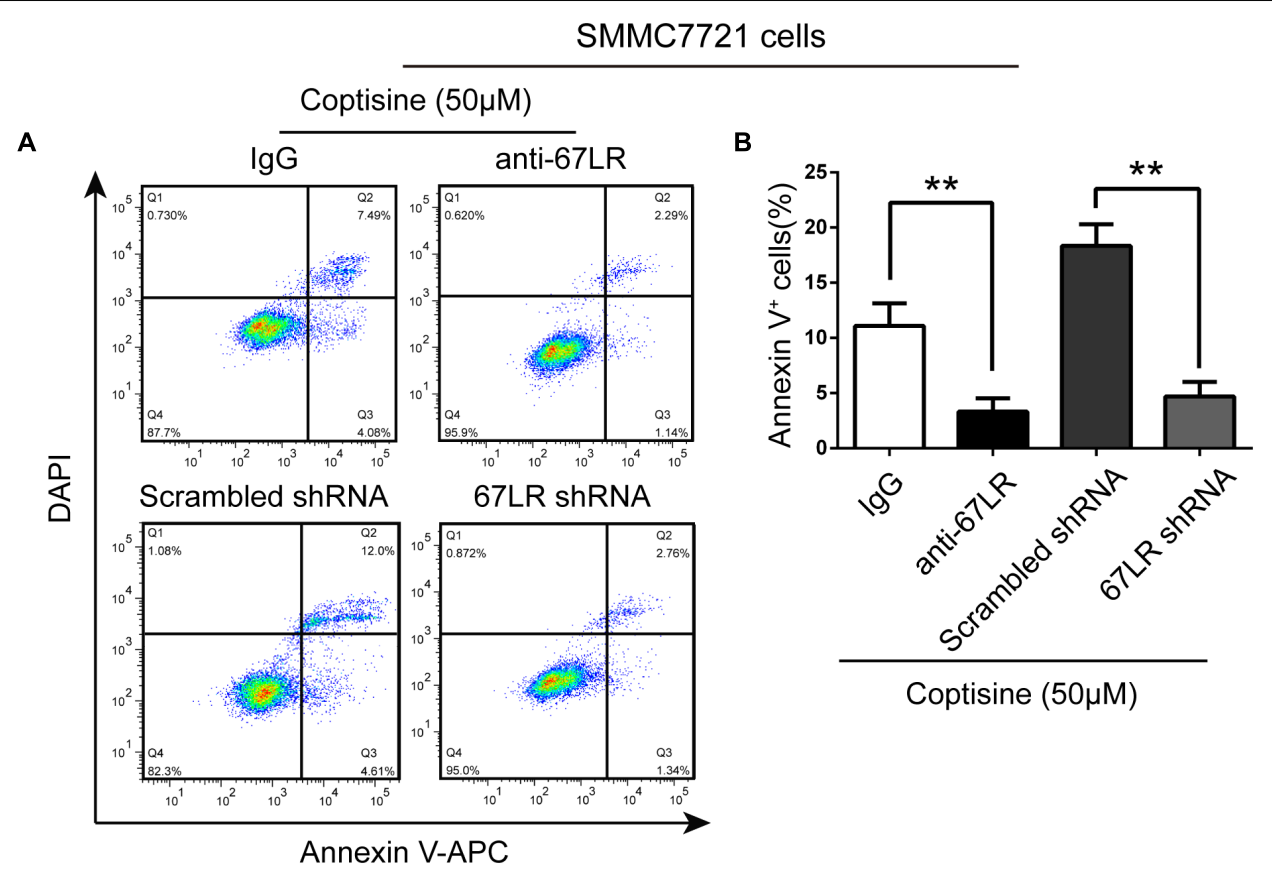

FIGURE 5 | 67LR activity significantly influenced the effect of coptisine on SMMC7721 cell apoptosis. (A,B) Apoptotic cells were quantified using flow cytometry after staining with Annexin V-APC and DAPI according to the indicated groups. Also, the percentages of apoptotic cells were counted. ${ }^{* *} P<0.01$ compared with the appointed group. Values represent mean \pm SD with three replicates. 


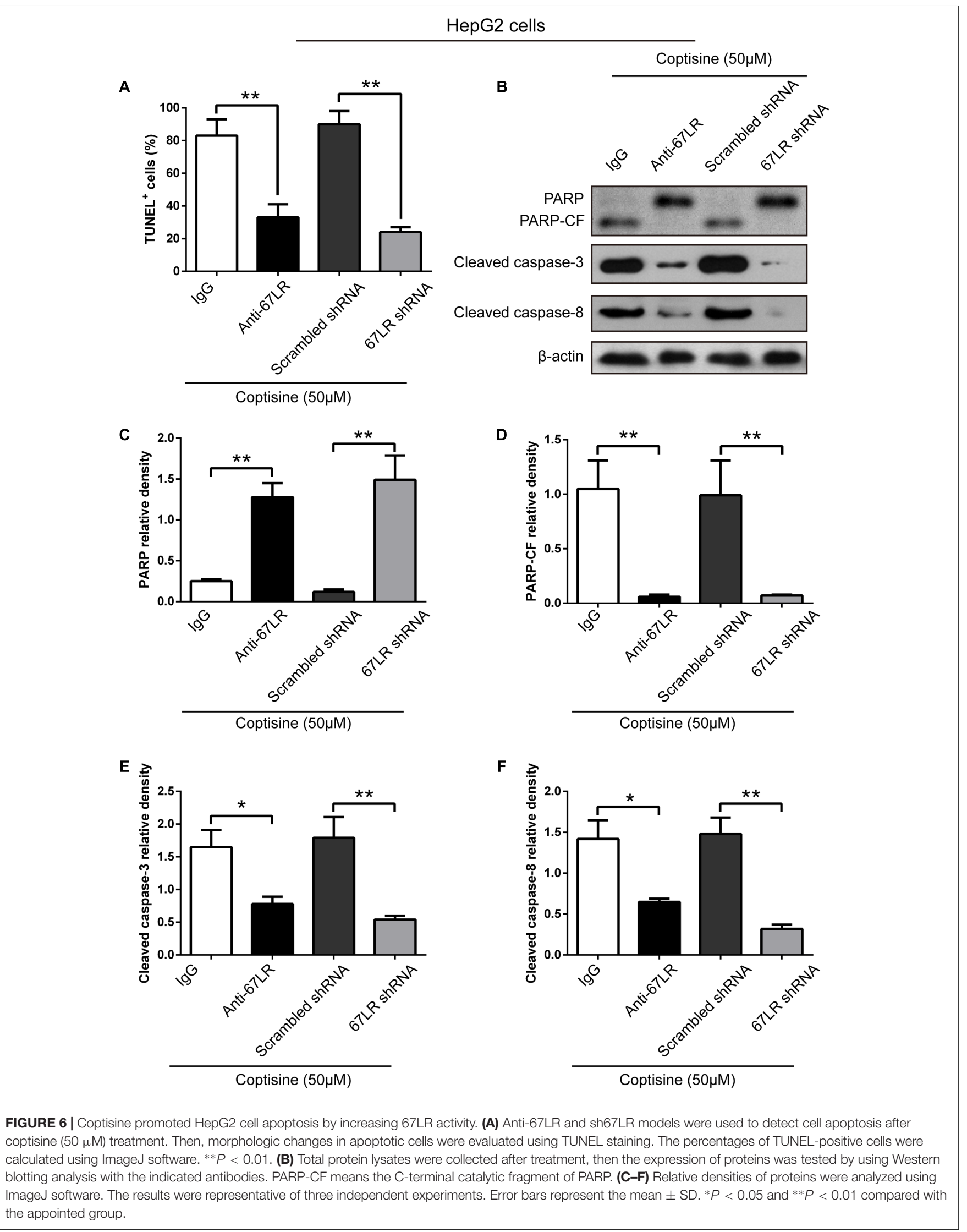


a rate-determining process of 67LR-dependent cell apoptosis. Furthermore, NS2028, a high-efficiency and high-specificity inhibitor of soluble guanylate cyclase, was confirmed to directly prevent the cGMP upregulation induced by 67LR agonist (Kumazoe et al., 2013a). The present study investigated, using NS2028 as the inhibitor, whether cGMP was involved in coptisine-induced apoptosis and inhibition of cell viability in SMMC7721 cells. The concentration of cGMP was measured after treatment with coptisine alone and then with a combination of coptisine with NS2028 by using an ELISA kit. Coptisine significantly enhanced the production of cGMP, which could be blocked by NS2028 treatment (Figure 8C). Low cytotoxicity was observed after NS2028 treatment alone, and NS2028 significantly reduced coptisine-induced inhibition of cell viability (Figure 7A). Western blot analysis revealed that NS2028 observably decreased coptisine-induced PARP cleavage and the expression of cleaved caspases 3 and 8 (Figures 7B-F). Moreover, flow cytometry results also showed that NS2028 markedly decreased the number of coptisine-induced apoptotic cells (Figures 8A,B). These results indicated that cGMP was involved in coptisine-induced apoptosis and inhibition of cell viability.
A

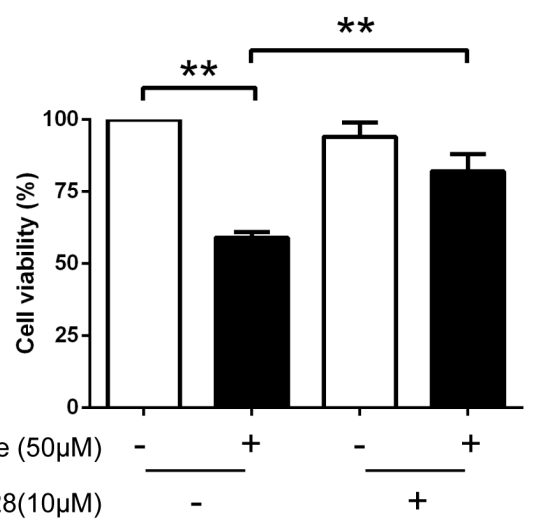

B
NS2028 $(10 \mu \mathrm{M})$
Coptisine $(50 \mu \mathrm{M})$

PARP

PARP-CF

Cleaved caspase-3

Cleaved caspase-8

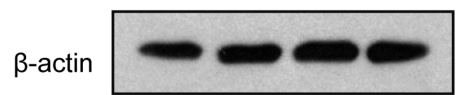

c

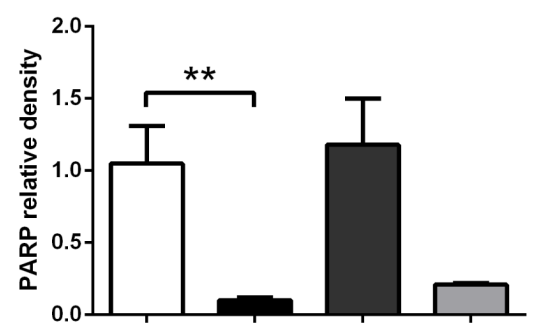

D

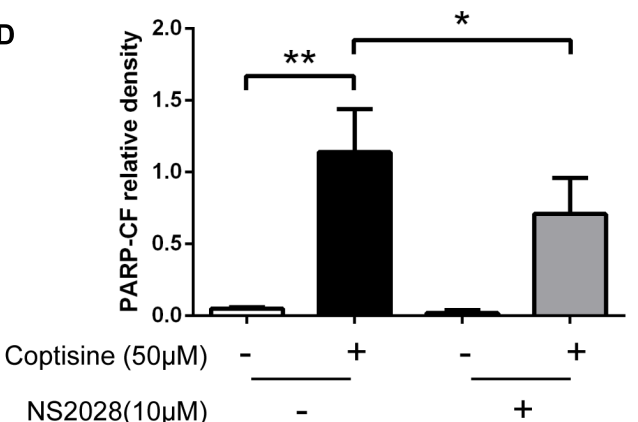

F

E

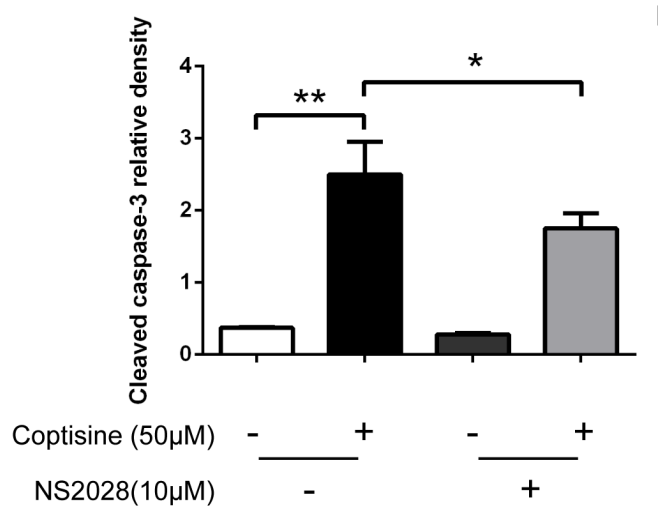

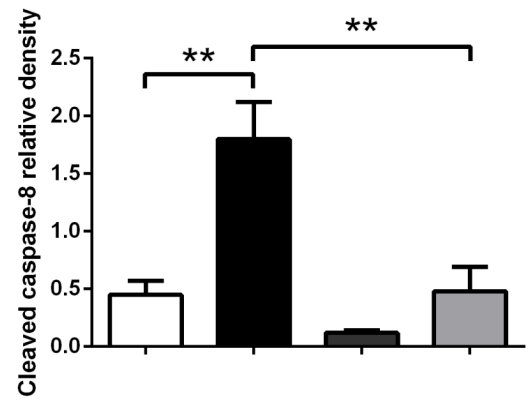

FIGURE 7 | Inhibiting the expression of cGMP could obviously reduce coptisine-induced apoptosis. (A) The viability of SMMC7721 cells was detected using CCK-8 assay according to the indicated groups. NS2028 (5 $\mu \mathrm{M})$ was used to inhibit the expression of cGMP. (B) Total protein lysates were collected after coptisine and (or) NS2028 treatment, and then the expression of proteins was detected using Western blot analysis using the indicated antibodies. PARP-CF means the C-terminal catalytic fragment of PARP. (C-F) The relative densities of these proteins were analyzed using Image software. ${ }^{*} P<0.05$ and ${ }^{* *} P<0.01$ compared with the appointed group ( $n=3$ per group for all the studies). All data are expressed as mean \pm SD. 


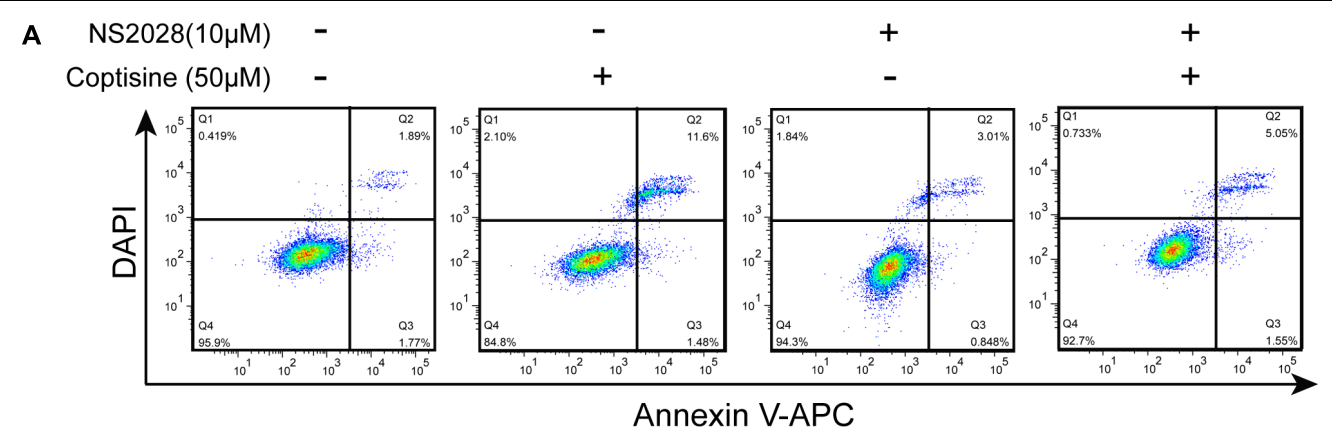

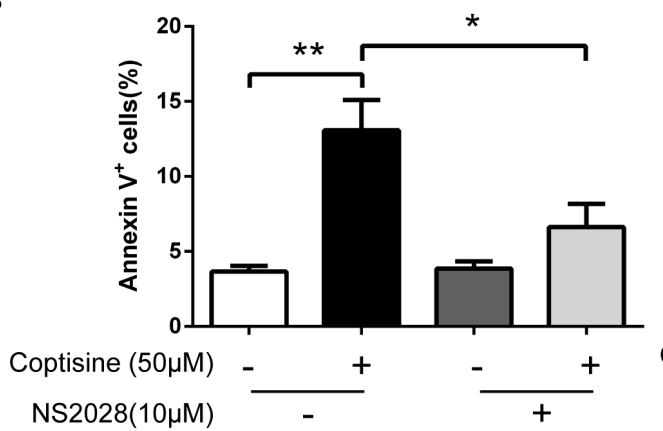

C

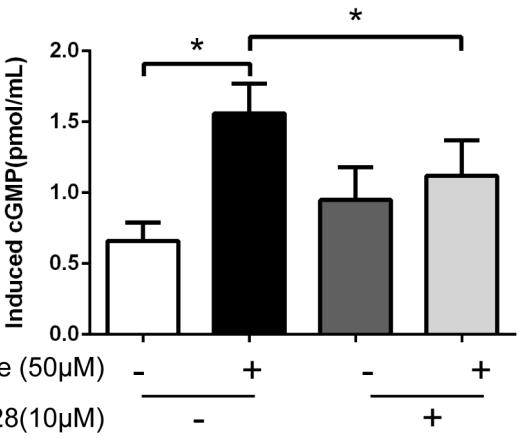

FIGURE 8 | Coptisine induced cGMP upregulation after activating 67LR in SMMC7721 cells. (A,B) Apoptotic cells were quantified using flow cytometry after staining with Annexin V-APC and DAPI according to the indicated groups. Also, the percentages of apoptotic cells were counted $(n=3)$. (C) The concentration of induced cGMP was measured using an ELISA kit after coptisine and (or) NS2028 treatment $(n=6){ }^{*} P<0.05$ and $* * P<0.01$ compared with the appointed group. All data are expressed as mean $\pm \mathrm{SD}$.

\section{Coptisine Inhibited Tumor Growth, Induced Apoptosis, and Activated 67LR/cGMP Signaling in an SMMC7721 Xenograft Model}

The cells were injected subcutaneously into male nude mice to evaluate the in vivo activity of coptisine on SMMC7721 and sh67LR SMMC7721 cells. After the appearance of palpable tumors, the mice were divided into five groups randomly: control, coptisine + IgG, coptisine + anti-67LR, coptisine + scramble shRNA, and coptisine + sh67LR. Then, the mice were injected intraperitoneally with normal saline, $50 \mathrm{mg} / \mathrm{kg}$ coptisine, and/or $20 \mu \mathrm{g} / \mathrm{mL}$ anti-67LR every day.

As shown in Figures 9A,B, the tumor growth was significantly suppressed 15 days after initiating coptisine treatment. These events became more apparent after 25 days of drug exposure. In addition, tumor volumes of the coptisine + sh67LR group were markedly higher than those of the coptisine + scramble shRNA group. Moreover, treatment with anti-67LR antibody significantly blocked coptisine-induced inhibition of tumor growth. However, no statistically significant changes in body weight were observed compared with each group (Figure 9C). The mice in the four coptisine treatment groups did not show any other signs of toxicity, such as agitation, indigestion or diarrhea, impaired movement or posture, and areas of redness or swelling. Western blot analysis was used to test the expression of apoptoticrelated proteins, including PARP and cleaved caspases 8 and 3 , so as to verify that 67LR was involved in coptisine-induced
SMMC7721 cell apoptosis. As shown in Figures 9D-H, the expression of C-terminal catalytic fragment of PARP and cleaved caspases 3 and 8 was significantly induced in the coptisine + IgG group, whereas treatment with coptisine + anti-67LR and coptisine + sh67LR resulted in an obvious decrease in the expression of these apoptotic -related proteins. Moreover, tumor samples in the coptisine + scramble shRNA and coptisine + sh67LR groups were excised, sectioned, and then analyzed using $\mathrm{H} \& \mathrm{E}$ staining and immunohistochemical analysis. As shown in Figure 10A, the results of H\&E staining showed that tumors in both coptisine + sh67LR and coptisine + scramble shRNA groups had no typical pathological appearance but inconspicuous inflammation, signs of necrosis, and fibrosis. The immunohistochemical analysis further revealed that mice treated with coptisine and sh67LR displayed decreased immunoreactivity for cleaved caspases 8 and 3, indicating that sh67LR blocked coptisine-induced cell apoptosis in the SMMC7721 xenograft model (Figure 10A). Above all, these findings indicated that coptisine significantly inhibited SMMC7721 xenograft growth without obvious side effects, and coptisine-induced cell apoptosis was associated with 67LR activation.

\section{DISCUSSION}

Recent studies showed that some isoquinoline alkaloids and their $\mathrm{N}$-oxides exhibited different pharmacological activities because of a potential structure-activity relationship (Iizuka et al., 2002; 
A

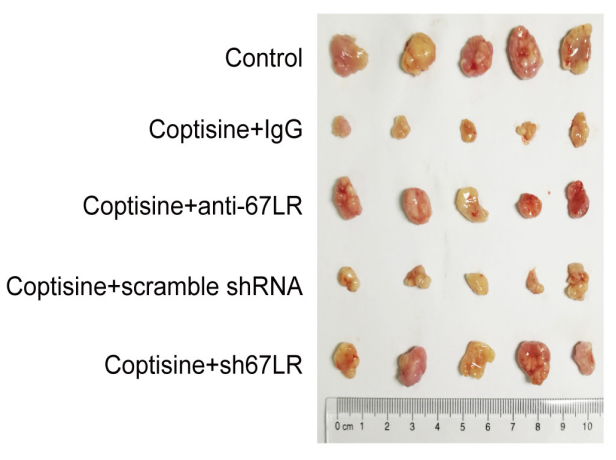

C

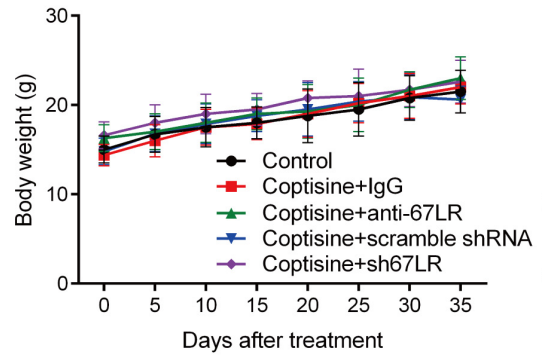

E

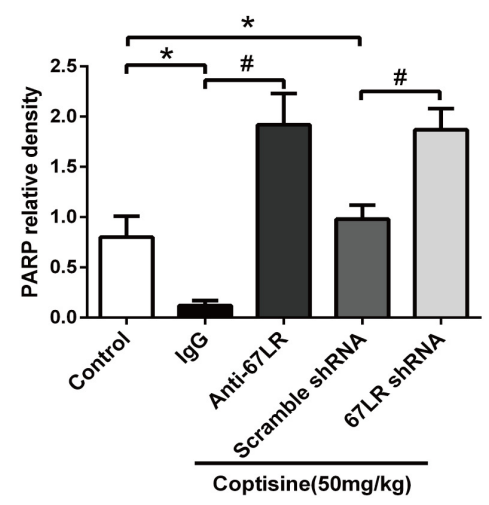

G

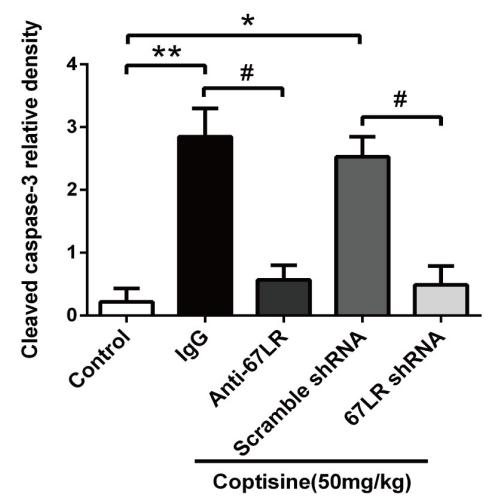

B

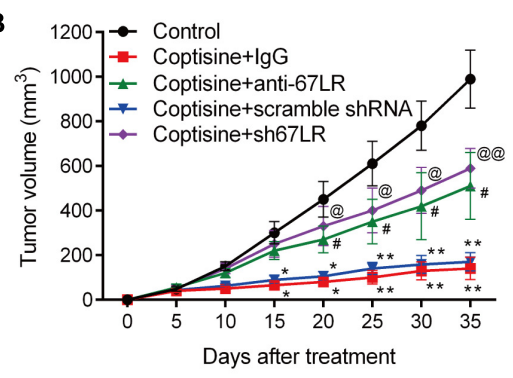

Coptisine $(50 \mathrm{mg} / \mathrm{kg})$

D

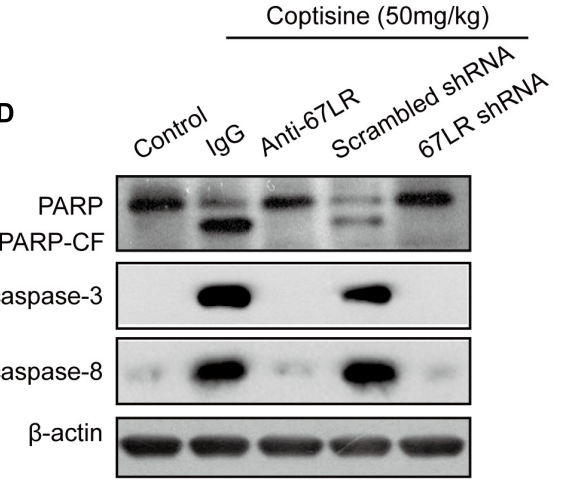

$\mathbf{F}$

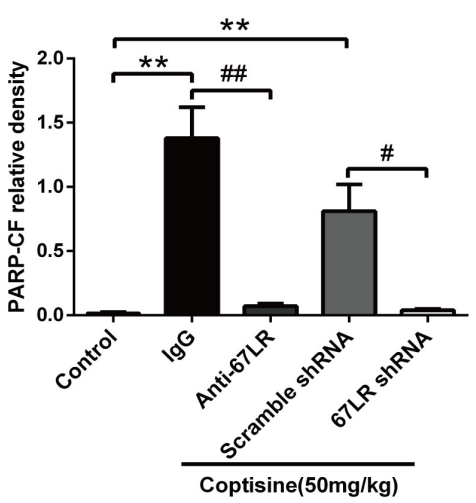

$\mathrm{H}$

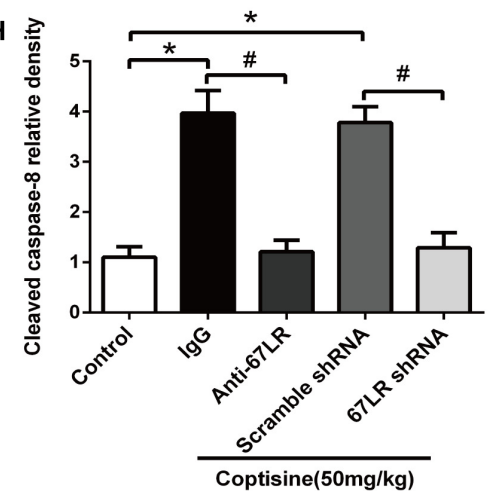

FIGURE 9 | Coptisine inhibited tumor growth and induced apoptosis in a SMMC7721 xenograft animal model. A total of 50 nude mice were inoculated with SMMC7721 cells and then randomly divided into 5 groups $(n=10)$. (A) Images were exhibited for five representative tumors from each group after 35 days of treatment. (B) Tumor volumes were measured according to the indicated intervals. Data are expressed as mean \pm SD. ${ }^{*} P<0.05$ and ${ }^{* *} P<0.01$ compared with the same-day results of the control group. ${ }^{\#} P<0.05$ compared with the same-day results of the coptisine $+\operatorname{lgG}$ group. ${ }^{\circledR} P<0.05$ and ${ }^{@ @ ~} P<0.01$ compared with the same-day results of the coptisine + scramble shRNA group. (C) Changes in the body weight of mice were recorded during the 35 days of treatment. (D-H) Tumors from all the five groups were lysed and collected to detect the expression of objective proteins using Western blot analysis. PARP-CF means the C-terminal catalytic fragment of PARP. The relative densities of these proteins were analyzed using ImageJ software. ${ }^{*} P<0.05,{ }^{* *} P<0.01$, and ${ }^{\#} P<0.05$ ( $n=3$ per group for all the studies. All data are expressed as mean \pm SD. 
A

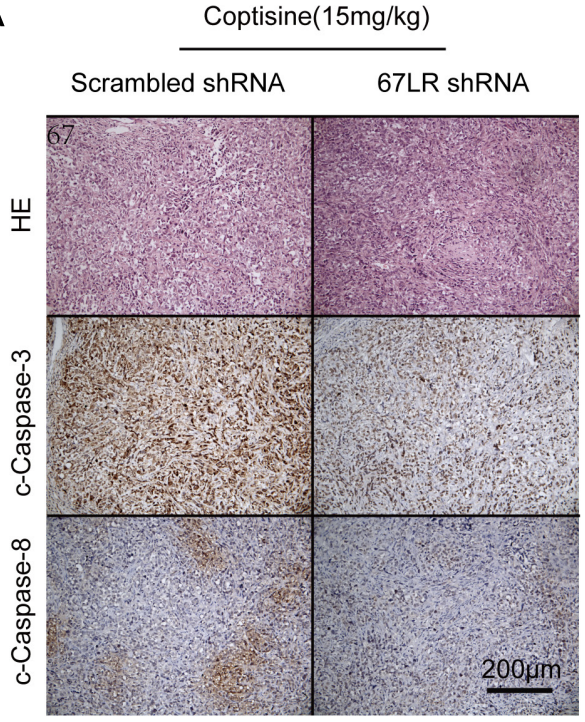

B

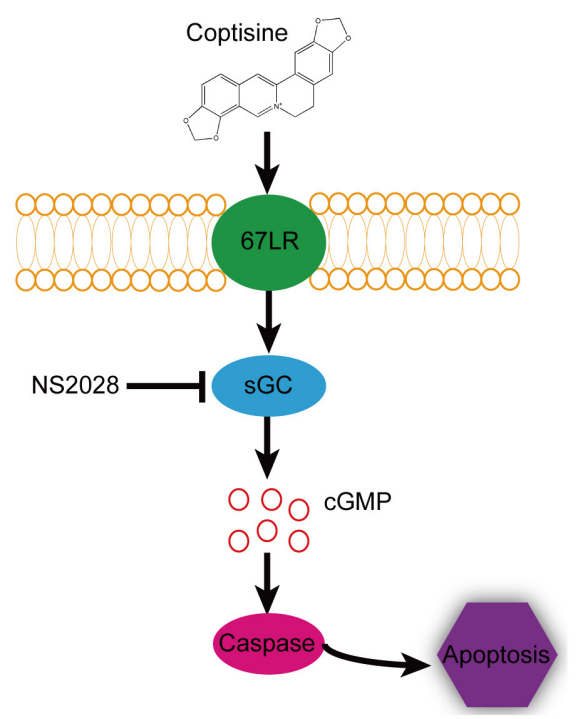

FIGURE 10 | Coptisine induced apoptosis through a 67LR-dependent pathway in a SMMC7721 xenograft animal model. (A) Tumors from the coptisine + scramble shRNA and coptisine + sh67LR groups were fixed and stained with H\&E to inspect tumor cell morphology. Also, immunohistochemical analysis was used to evaluate the levels of apoptotic -related proteins. Scale bar represents $200 \mu \mathrm{m}$. (B) An illustration of the molecular mechanism of coptisine-induced apoptosis. Coptisine induced 67LR functional activation and promoted sGC conversion to generate cGMP, resulting in cGMP upregulation, then caspase activation, and finally apoptosis.

Yu et al., 2014). Coptisine, one of the isoquinoline alkaloids, has been reported to exhibit an anti-tumor effect on colon and breast cancer, but little is known regarding its effect on liver cancer (Zhang et al., 2014; Li et al., 2017). Tao et al. reported that the anti-tumor property of coptisine was comparable and even exceeded that of the conventional anti-tumor drugs such as cisplatin and adriamycin, which was similar to the inhibition of the growth of tumor xenografts in a mouse model (Huang T. et al., 2017). Our results demonstrated that coptisine selectively induced apoptosis in SMMC7721, HepG2 and BEL7402 cells (HCC cell lines) and inhibited the tumor growth in xenografts without obvious toxicity, suggesting that coptisine could be developed into a novel anti-tumor agent for treating liver cancer.

The present study, using a variety of techniques and different perspectives, demonstrated that coptisine significantly inhibited cell viability and induced apoptosis in HCC cells but not in LO2 cells. On the one hand, the CCK-8 assay was used to confirm that coptisine significantly decreased cell viability under the influence of mitochondrial dehydrogenase; the number of generated formazan was proportional to the number of living cells. On the other hand, the TUNEL technology was used to verify coptisine-induced cell apoptosis in a concentration-dependent manner through DNA damage. Annexin V-APC/DAPI staining intuitively distinguished the apoptotic cells by labeling them with fluorescent probes. Additionally, the detection of apoptotic-related proteins, including PARP and caspases 8 and 3, by Western blot analysis suggested that caspase-dependent apoptotic pathways were triggered in coptisine-treated HCC cells. Above all, coptisine could induce apoptosis in a large number of cells, but the molecular mechanism underlying coptisine-induced apoptosis needed further investigation.

The 67-kDa laminin receptor (67LR) has been used as a cancer-specific death receptor in a caspase-dependent cell death receptor pathway. $67 \mathrm{LR}$, derived from a $37-\mathrm{kDa}$ laminin receptor precursor, is a non-integrin cell surface receptor located in the cell membrane for extracellular matrix binding with high-affinity laminin-1 (Dorchies et al., 2009; Jovanovic et al., 2015). Recent studies revealed that 67LR was overexpressed in neoplastic cells and associated with an increased invasion and metastasis in human solid tumors (Pesapane et al., 2017). Moreover, 67LR is confirmed to be a new promising target for cancer treatment in some cancer cells (Mocanu et al., 2014; Lu et al., 2016). Kumazoe et al. (2013b) reported that when 67LR together with its natural ligand EGCG was activated, it in turn activated a signal transduction pathway, thus inhibiting growth and inducing apoptosis in cancer cells without detrimentally affecting the normal cells (Kumazoe et al., 2013a,b). Therefore, 67LR, as a new cancer-specific death receptor, promoted cell apoptosis.

This study demonstrated the effect of 67LR on coptisineinduced cell apoptosis. Concretely, 67LR itself was highly expressed in SMMC7721 cells rather than in normal LO2 cells. Also, 67LR-neutralizing antibody or sh67LR could significantly reduce the apoptotic effect of coptisine both in vivo and in vitro, suggesting that 67LR participated in coptisine-induced apoptosis of liver cancer. In addition, the protein expression of caspases 8 and 3 and PARP in the downstream of cell death receptor pathway was totally influenced by 67LR. Taken together, the results of the present study were consistent with the findings of other studies and indicated that 67LR activation contributed to the coptisine-induced apoptosis. 
The findings also provided evidence that a 67LR/cGMP signaling pathway was involved in coptisine-induced apoptosis. Cyclic guanosine $3^{\prime}, 5^{\prime}$-monophosphate (cGMP) is generated by guanylate cyclase. It is an omnipresent second messenger responsible for transducing extracellular signaling pathways. It has been confirmed to be involved in regulating various physiological functions such as platelet aggregation, neurotransmission, and vascular smooth muscle regulation (Saravani et al., 2012). Besides, studies reported that cGMP was crucial in cell proliferation, differentiation, and apoptosis (Tsukamoto et al., 2015; Huang Y. et al., 2017). Particularly, cGMP was confirmed to be an important signaling molecule downstream of 67LR. The cGMP upregulation could exactly be a rate-determining process of 67LR-dependent cell apoptosis (Tsukamoto et al., 2012; Kumazoe et al., 2013a; Montuori et al., 2016). NS2028, a high-efficiency and high-specificity inhibitor of soluble guanylate cyclase, was confirmed to directly prevent the cGMP upregulation induced by 67LR agonist (Kumazoe et al., 2013a,b). The study also showed that cGMP was important in coptisine-induced apoptosis. Concretely, the cell viability significantly increased when the cells were treated with both coptisine and NS2028 compared with only coptisine. Also, the expression of apoptotic -related proteins obviously reduced after the cells were exposed to coptisine and NS2028. Even more intuitively, the concentration of cGMP in SMMC7721 cells increased with coptisine treatment, but it was inhibited on exposure to both coptisine and NS2028. Therefore, coptisine induced apoptosis in SMMC7721 cells probably through a $67 \mathrm{LR}-\mathrm{cGMP}$-caspase 3 pathway. In addition, coptisine possessed the function of the anti-inflammation and antioxidant stress in non-tumor cells. Wu et al. (2016) found that coptisine suppressed macrophage inflammatory responses by inhibiting phosphorylation c-Jun NH2-terminal kinase (JNK), p38 mitogen-activated protein kinase (MAPK), and phosphoinositide 3-kinase/Akt (PI3K/Akt). However, coptisine against AAPH induced oxidative stress by activating Akt and JNK (Hu et al., 2017). Therefore, coptisine shows different effects on the same signaling pathway in different cells and models, which opportunely shows its multiple biological functions.

We noticed that the expression of 67-LR was higher in cancer cells than in normal hepatic cells, which was also verified by flow cytometry showing that fluorochrome-labeled coptisine binds to cancer cells with higher affinity than to normal cells. Flow cytometry analysis also showed that 67-LR is expressed on the surface of the T-cell lymphoma line H9. This is relevant since another death receptor, CD95, is expressed on the surface of human lymphocytes and the interaction of CD95 with its respective ligand (Fas ligand) results in cell apoptosis, however,

\section{REFERENCES}

Boege, Y., Malehmir, M., Healy, M. E., Bettermann, K., Lorentzen, A., and Vucur, M. (2017). A dual role of caspase-8 in triggering and sensing proliferation-associated DNA damage, a key determinant of liver cancer development. Cancer Cell 32, 342.e10-359.e10. doi: 10.1016/j.ccell.2017. 08.010 since in this study we did not investigate the expression of 67-LR in primary human lymphocytes, it is unknown whether Coptisine induces apoptosis or activation of normal human immune cells.

\section{CONCLUSION}

This novel study indicated that coptisine effectively induced death receptor-dependent apoptosis in HCC cells and inhibited tumor growth of xenografts by activating 67LR/cGMP signaling. Collectively, the results supported a hypothetical model of coptisine-induced apoptosis in HCC cells (Figure 10B). In this model, coptisine induced 67LR activation and led to cGMP upregulation, resulting in caspase $8 / 3$ activation and, finally, apoptosis. Further efforts to explore the mechanism underlying coptisine-induce apoptosis through a 67LR/cGMP signaling pathway might guide the efficient treatment of liver cancer and other hepatic malignancies.

\section{ETHICS STATEMENT}

The university's institutional animal care and use committee approved all the animal studies.

\section{AUTHOR CONTRIBUTIONS}

LZ, FY, GL, YL, and QZ carried out the experiments. LZ, QT, $\mathrm{CH}$, and $\mathrm{JH}$ analyzed the data. LZ, FY, and RZ designed the experiments. LZ and RZ wrote the paper.

\section{FUNDING}

This work was supported by the National Natural Science Foundation of China (Nos. 81703481 and 31600806) and fund support in the General Hospital of Tibet Military (Y201710).

\section{SUPPLEMENTARY MATERIAL}

The Supplementary Material for this article can be found online at: https://www.frontiersin.org/articles/10.3389/fphar. 2018.00517/full\#supplementary-material

FIGURE S1 | Verification of lentiviral gene transfer. (A) The Lentiviral vector carrying sh67LR gene was constructed. (B) Red fluorescence (mCherry) expressing sh67LR plasmids successfully expressed in SMMC7721 cells. Scale bar represents $200 \mu \mathrm{m}$.

Dorchies, O. M., Wagner, S., Buetler, T. M., and Ruegg, U. T. (2009). Protection of dystrophic muscle cells with polyphenols from green tea correlates with improved glutathione balance and increased expression of 67LR, a receptor for (-)-epigallocatechin gallate. Biofactors 35, 279-294. doi: 10.1002/ biof.34

Fan, L., Xiao, Q., Chen, Y., Chen, G., Duan, J., and Tao, W. (2017). Pekinenin $\mathrm{E}$ inhibits the growth of hepatocellular carcinoma by promoting endoplasmic 
reticulum stress mediated cell death. Front. Pharmacol. 8:424. doi: 10.3389/ fphar.2017.00424

Gravitz, L. (2014). Liver cancer. Nature 4:516. doi: 10.1038/516S1a

Hu, Y. R., Ma, H., Zou, Z. Y., He, K., Xiao, Y. B., Wang, Y., et al. (2017). Activation of Akt and JNK/Nrf2/NQO1 pathway contributes to the protective effect of coptisine against AAPH-induced oxidative stress. Biomed. Pharmacother. 85, 313-322. doi: 10.1016/j.biopha.2016.11.031

Huang, T., Xiao, Y., Yi, L., Li, L., Wang, M., Tian, C., et al. (2017). Coptisine from Rhizoma Coptidis suppresses HCT-116 cells-related tumor growth in vitro and in vivo. Sci. Rep. 7:38524. doi: 10.1038/srep38524

Huang, W., Chen, Z., Zhang, L., Tian, D., Wang, D., Fan, D., et al. (2015). Interleukin-8 Induces Expression of FOXC1 to promote transactivation of CXCR1 and CCL2 in hepatocellular carcinoma cell lines and formation of metastases in mice. Gastroenterology 149, 1053.e14-1067.e14. doi: 10.1053/j. gastro.2015.05.058

Huang, W., Yuan, X., Sun, T., Fan, S., Wang, J., Zhou, Q., et al. (2017). Proteasome Inhibitor YSY01A abrogates constitutive STAT3 signaling via down-regulation of Gp130 and JAK2 in human A549 lung cancer cells. Front. Pharmacol. 8:476. doi: 10.3389/fphar.2017.00476

Huang, Y., Sumida, M., Kumazoe, M., Sugihara, K., Suemasu, Y., Yamada, S., et al. (2017). Oligomer formation of a tea polyphenol, EGCG, on its sensing molecule $67 \mathrm{kDa}$ laminin receptor. Chem. Commun. 53, 1941-1944. doi: 10.1039/c6cc09504f

Iizuka, K. (2016). The transcription factor carbohydrate-response elementbinding protein (ChREBP): a possible link between metabolic disease and cancer. Biochim. Biophys. Acta 1863, 474-485. doi: 10.1016/j.bbadis.2016. 11.029

Iizuka, N., Hazama, S., Yoshimura, K., Yoshino, S., Tangoku, A., Miyamoto, K., et al. (2002). Anticachectic effects of the natural herb Coptidis rhizoma and berberine on mice bearing colon 26/clone 20 adenocarcinoma. Int. J. Cancer 99, 286-291. doi: 10.1002/ijc.10338

Jin, H., Wang, C., Jin, G., Ruan, H., Gu, D., Wei, L., et al. (2017). Regulator of calcineurin 1 Gene Isoform 4, down-regulated in hepatocellular carcinoma, prevents proliferation, migration, and invasive activity of cancer cells and metastasis of orthotopic tumors by inhibiting nuclear translocation of NFAT1. Gastroenterology 153, 799.e33-811.e33. doi: 10.1053/j.gastro.2017. 05.045

Jovanovic, K., Chetty, C. J., Khumalo, T., Da Costa Dias, B., Ferreira, E., Malindisa, S. T., et al. (2015). Novel patented therapeutic approaches targeting the $37 / 67 \mathrm{kDa}$ laminin receptor for treatment of cancer and Alzheimer's disease. Expert Opin. Ther. Pat. 25, 567-582. doi: 10.1517/13543776.2015. 1014802

Kou, S., Han, B., Wang, Y., Huang, T., He, K., Han, Y., et al. (2016). Synergetic cholesterol-lowering effects of main alkaloids from Rhizoma Coptidis in HepG2 cells and hypercholesterolemia hamsters. Life Sci. 151, 50-60. doi: 10.1016/j.lfs. 2016.02.046

Kumazoe, M., Kim, Y., Bae, J., Takai, M., Murata, M., Suemasu, Y., et al. (2013a). Phosphodiesterase 5 inhibitor acts as a potent agent sensitizing acute myeloid leukemia cells to $67-\mathrm{kDa}$ laminin receptor-dependent apoptosis. FEBS Lett. 587, 3052-3057. doi: 10.1016/j.febslet.2013.07.041

Kumazoe, M., Sugihara, K., Tsukamoto, S., Huang, Y., Tsurudome, Y., Suzuki, T., et al. (2013b). $67-\mathrm{kDa}$ laminin receptor increases cGMP to induce cancer-selective apoptosis. J. Clin. Invest. 123, 787-799. doi: 10.1172/jci 64768

Kwon, H. A., Kwon, Y. J., Kwon, D. Y., and Lee, J. H. (2008). Evaluation of antibacterial effects of a combination of Coptidis Rhizoma, Mume Fructus, and Schizandrae Fructus against Salmonella. Int. J. Food Microbiol. 127, 180-183. doi: 10.1016/j.ijfoodmicro.2008.06.020

Li, G., Zhou, J., Budhraja, A., Hu, X., Chen, Y., Cheng, Q., et al. (2015). Mitochondrial translocation and interaction of cofilin and Drp1 are required for erucin-induced mitochondrial fission and apoptosis. Oncotarget 6, 1834-1849. doi: 10.18632/oncotarget.2795

Li, Y., Zhou, Y., Si, N., Han, L., Ren, W., Xin, S., et al. (2017). Comparative metabolism study of five protoberberine alkaloids in liver microsomes from rat, rhesus monkey, and human. Planta Med. 83, 1281-1288. doi: 10.1055/s-0043108249

Lin, C. C., Ng, L. T., Hsu, F. F., Shieh, D. E., and Chiang, L. C. (2004). Cytotoxic effects of Coptis chinensis and Epimedium sagittatum extracts and their major constituents (berberine, coptisine and icariin) on hepatoma and leukaemia cell growth. Clin. Exp. Pharmacol. Physiol. 31, 65-69.

Lu, C. L., Xu, J., Yao, H. J., Luo, K. L., Li, J. M., Wu, T., et al. (2016). Inhibition of human $67-\mathrm{kDa}$ laminin receptor sensitizes multidrug resistance colon cancer cell line SW480 for apoptosis induction. Tumour Biol. 37, 1319-1325. doi: 10.1007/s13277-015-3873-5

Lu, X. L., Zeng, J., Chen, Y. L., He, P. M., Wen, M. X., Ren, M. D., et al. (2013). Sinomenine hydrochloride inhibits human hepatocellular carcinoma cell growth in vitro and in vivo: involvement of cell cycle arrest and apoptosis induction. Int. J. Oncol. 42, 229-238. doi: 10.3892/ijo.2012. 1704

Lv, L., Chen, G., Zhou, J., Li, J., and Gong, J. (2015). WT1-AS promotes cell apoptosis in hepatocellular carcinoma through down-regulating of WT1. J. Exp. Clin. Cancer Res. 34:119. doi: 10.1186/s13046-015-0233-7

Mocanu, M. M., Ganea, C., Georgescu, L., Varadi, T., Shrestha, D., Baran, I., et al. (2014). Epigallocatechin 3-O-gallate induces $67 \mathrm{kDa}$ laminin receptor-mediated cell death accompanied by downregulation of ErbB proteins and altered lipid raft clustering in mammary and epidermoid carcinoma cells. J. Nat. Prod. 77, 250-257. doi: 10.1021/np4007712

Montuori, N., Pesapane, A., Giudice, V., Serio, B., Rossi, F. W., De Paulis, A., et al. (2016). $67 \mathrm{kDa}$ laminin receptor (67LR) in normal and neoplastic hematopoietic cells: is its targeting a feasible approach? Transl. Med. UniSa 15, 8-14.

Pesapane, A., Ragno, P., Selleri, C., and Montuori, N. (2017). Recent advances in the function of the $67 \mathrm{kDa}$ laminin receptor and its targeting for personalized therapy in cancer. Curr. Pharm. Des. 23, 4745-4757. doi: 10.2174/ 1381612823666170710125332

Rao, P. C., Begum, S., Sahai, M., and Sriram, D. S. (2017). Coptisineinduced cell cycle arrest at $\mathrm{G} 2 / \mathrm{M}$ phase and reactive oxygen speciesdependent mitochondria-mediated apoptosis in non-small-cell lung cancer A549 cells. Tumour Biol. 39:1010428317694565. doi: 10.1177/10104283176 94565

Saravani, R., Karami-Tehrani, F., Hashemi, M., Aghaei, M., and Edalat, R. (2012). Inhibition of phosphodiestrase 9 induces cGMP accumulation and apoptosis in human breast cancer cell lines, MCF-7 and MDA-MB-468. Cell Prolif. 45, 199-206. doi: 10.1111/j.1365-2184.2012.00819.x

Song, T., Choi, C. H., Cho, Y. J., Sung, C. O., Song, S. Y., Kim, T. J., et al. (2012). Expression of $67-\mathrm{kDa}$ laminin receptor was associated with tumor progression and poor prognosis in epithelial ovarian cancer. Gynecol. Oncol. 125, 427-432. doi: 10.1016/j.ygyno.2012.01.030

Tsukamoto, S., Hirotsu, K., Kumazoe, M., Goto, Y., Sugihara, K., Suda, T., et al. (2012). Green tea polyphenol EGCG induces lipid-raft clustering and apoptotic cell death by activating protein kinase Cdelta and acid sphingomyelinase through a $67 \mathrm{kDa}$ laminin receptor in multiple myeloma cells. Biochem. J. 443, 525-534. doi: 10.1042/bj20111837

Tsukamoto, S., Huang, Y., Kumazoe, M., Lesnick, C., Yamada, S., Ueda, N., et al. (2015). Sphingosine Kinase-1 protects multiple myeloma from apoptosis driven by cancer-specific inhibition of RTKs. Mol. Cancer Ther. 14, 2303-2312. doi: 10.1158/1535-7163.mct-15-0185

Wu, J., Zhang, H., Hu, B., Yang, L., Wang, P., Wang, F., et al. (2016). Coptisine from Coptis chinensis inhibits production of inflammatory mediators in lipopolysaccharide-stimulated RAW 264.7 murine macrophage cells. Eur. J. Pharmacol. 780, 106-114. doi: 10.1016/j.ejphar.2016.03.037

Xu, D., Jin, J., Yu, H., Zhao, Z., Ma, D., Zhang, C., et al. (2017). Chrysin inhibited tumor glycolysis and induced apoptosis in hepatocellular carcinoma by targeting hexokinase-2. J. Exp. Clin. Cancer Res. 36:44. doi: 10.1186/s13046017-0514-4

Yang, F., Zhou, L., Qian, X., Wang, D., He, W. J., Tang, Z. W., et al. (2017). Adropin is a key mediator of hypoxia induced anti-dipsogenic effects via TRPV4CamKK-AMPK signaling in the circumventricular organs of rats. Front. Mol. Neurosci. 10:105. doi: 10.3389/fnmol.2017.00105

Yoshimoto, S., Loo, T. M., Atarashi, K., Kanda, H., Sato, S., Oyadomari, S., et al. (2013). Obesity-induced gut microbial metabolite promotes liver cancer through senescence secretome. Nature 499, 97-101. doi: 10.1038/nature 12347

Yu, D., Fu, S., Cao, Z., Bao, M., Zhang, G., Pan, Y., et al. (2014). Unraveling the novel anti-osteosarcoma function of coptisine and its mechanisms. Toxicol. Lett. 226, 328-336. doi: 10.1016/j.toxlet.2014.02.021 
Zhang, L. L., Ma, L. N., Yan, D., Zhang, C. E., Gao, D., Xiong, Y., et al. (2014). Dynamic monitoring of the cytotoxic effects of protoberberine alkaloids from Rhizoma Coptidis on HepG2 cells using the xCELLigence system. Chin. J. Nat. Med. 12, 428-435. doi: 10.1016/s1875-5364(14) 60067-4

Zhang, Y., Qiao, R., He, D., Zhao, Z., Yang, S., Zou, H., et al. (2016). Indazolo[3,2-b]quinazolinones attack hepatocellular carcinoma Hep3B cells by inducing mitochondrial-dependent apoptosis and inhibition of Nrf2/ARE signaling pathway. Curr. Mol. Med. 16, 820-828. doi: 10.2174/1566524016666161128114444

Zhou, L., Zheng, Y., Li, Z., Bao, L., Dou, Y., Tang, Y., et al. (2016). Compound K attenuates the development of atherosclerosis in ApoE(-/-) Mice via LXRalpha activation. Int. J. Mol. Sci. 8:E1054. doi: 10.3390/ijms17071054

Zhou, Y., Yu, Q., Qin, X., Bhavsar, D., Yang, L., Chen, Q., et al. (2016). Improving the anticancer efficacy of laminin receptor-specific therapeutic ruthenium nanoparticles (RuBB-Loaded EGCG-RuNPs) via ROS-Dependent apoptosis in SMMC-7721 Cells. ACS Appl. Mater. Interfaces 8, 15000-15012. doi: 10.1021/ acsami.5b02261

Conflict of Interest Statement: The authors declare that the research was conducted in the absence of any commercial or financial relationships that could be construed as a potential conflict of interest.

Copyright (c) 2018 Zhou, Yang, Li, Huang, Liu, Zhang, Tang, Hu and Zhang. This is an open-access article distributed under the terms of the Creative Commons Attribution License (CC BY). The use, distribution or reproduction in other forums is permitted, provided the original author(s) and the copyright owner are credited and that the original publication in this journal is cited, in accordance with accepted academic practice. No use, distribution or reproduction is permitted which does not comply with these terms. 